\title{
The economic effect of the implementation of the differential pressure in the gas distribution
}

\author{
Viktor Zhila, and Aleksey Klochko* \\ Moscow State University of Civil Engineering, Yaroslavskoe shosse, 26, Moscow, 129337, Russia
}

\begin{abstract}
This paper demonstrates the problem of calculating a rational piezometric pressure distribution graph in high-and medium-pressure gas distribution networks. These issues are particularly relevant in the design of gas distribution networks. In addition to the often used in practice design opportunities to reduce the cost of building gas distribution networks, rational choice of the optimal configuration of gas pipelines, which gives us the opportunity to get a large economic, as well as the effect of preserving the environment, it is also possible to use the pressure drop in the network. In total, with a rational choice of the scheme of organizing gas supply to consumers in localities, it is possible to get the maximum economic effect. It is important that modern equipment of this type allows to implement a single-stage scheme of gas distribution networks. Construction of natural gas pipelines we can use the available pressure drop in various technical solutions, but the reasonable distribution of the pressure drop between the network sections allows us to obtain a positive economic effect. The algorithm shown is proposed for use in the design of gas distribution networks of settlements of all types, and in the supply of natural gas to industrial enterprises.
\end{abstract}

\section{Introduction}

At the branched gas networks of medium and high pressure, gas is supplied to consumers in one section. Consumers have one-way power supply, so the transit costs are determined unambiguously, therefore, the estimated costs for all sections are known. Medium and high pressure distribution systems rely on a constant calculation of the pressure drop. The pressure losses between the successive sections are distributed in such a way that the total cost of the distribution system should be minimal [1].

\section{Literature review}

The gas distribution scheme becomes flexible: independence of connection of any object is achieved, metal consumption is reduced, investments in construction are reduced by reducing the diameter of gas pipelines. In this case, the distribution gas network is completely designed for medium pressure. When choosing a dead-end gas network of

\footnotetext{
*Corresponding author: klo4ko_aleksey@mail.ru
} 
medium pressure, it is necessary to make a technical and economic calculation, in which it is possible to determine the optimal shape of the piezometer, which will contribute to achieving the minimum cost of the gas distribution network.

The choice of the optimal pressure drop for dead-end networks is considered in the works [2,3], but these recommendations and algorithms affect and are applicable only for low-pressure networks, and there are no recommendations for choosing the optimal form of the piezometric pressure drop graph for high-and medium-pressure networks. In this paper, we will consider this issue. The classical scheme of pressure distribution in a dead-end gas distribution network is shown in Figure 1

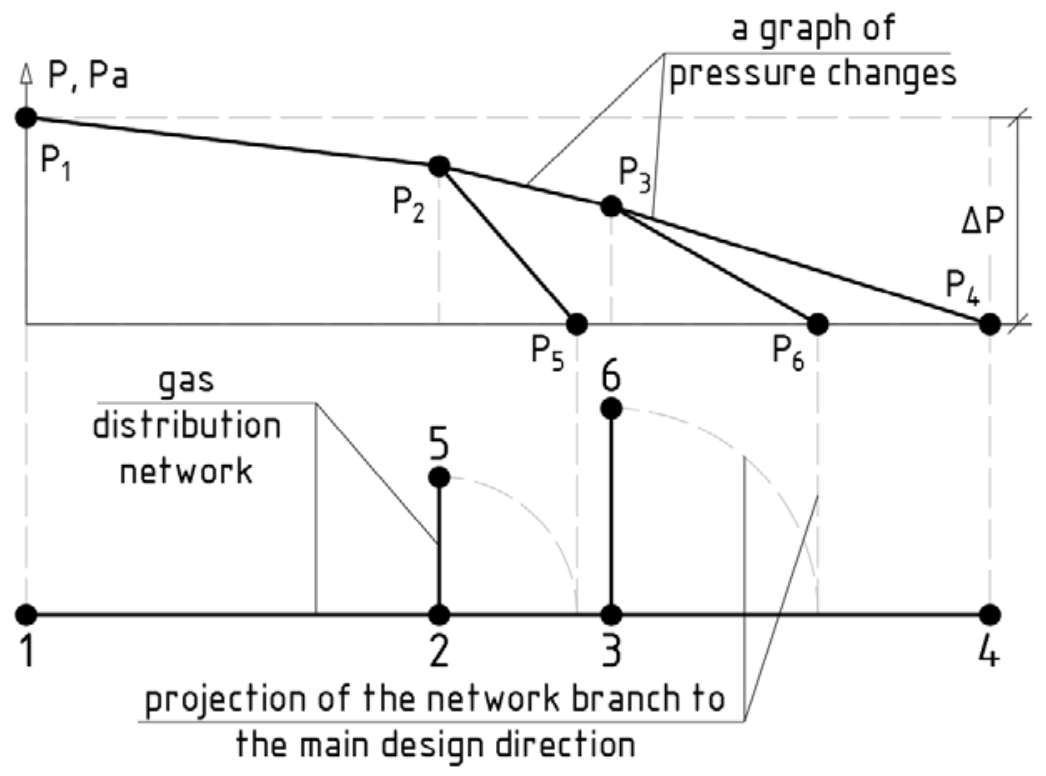

Fig. 1. Pressure change in the gas pipeline

\section{Materials and methods}

Optimization of gas distribution networks is an urgent task, which is devoted to many works [4 - 24]. The method of rational distribution of pressure between the sections of the gas distribution network in its design was developed at the Moscow State University of Civil Engineering at the Department of Heat Supply, Gas Supply and Ventilation. Consider the application of the method of rational distribution of the pressure drop in the gas distribution network, which we have when designing the network by the example of choosing the optimal option for a dead-end gas distribution system shown in Figure 2 


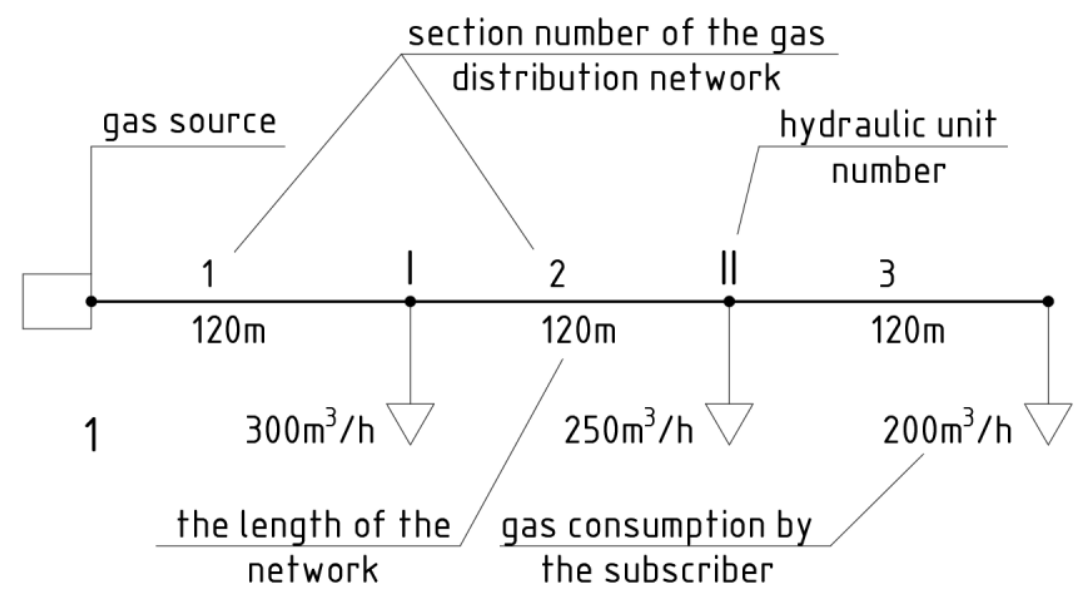

Fig. 2. Calculated scheme of the high-pressure network

The results of the preliminary hydraulic calculation of the high-pressure network are presented in Table 1.

Table 1. Preliminary hydraulic calculation of the high-pressure network.

\begin{tabular}{|c|c|c|c|c|}
\hline $\begin{array}{c}\text { Network } \\
\text { section } \\
\text { number }\end{array}$ & $\begin{array}{c}\text { Length of the } \\
\text { network section, } \\
\mathbf{m}\end{array}$ & $\begin{array}{c}\text { Calculated gas } \\
\text { consumption, m3 / } \\
\mathbf{h}\end{array}$ & $\begin{array}{c}\text { pipe size } \\
\boldsymbol{d} \times \boldsymbol{s}, \\
\mathbf{m m}\end{array}$ & $\begin{array}{c}\text { Pressure drop, } \\
\mathbf{k P a}\end{array}$ \\
\hline 1 & 120 & 750 & $90 \times 3$ & 1588 \\
\hline 2 & 120 & 450 & $70 \times 3$ & 2008 \\
\hline 3 & 120 & 200 & $50 \times 3$ & 2133,8 \\
\hline
\end{tabular}

\section{Results of research}

The gas pressure loss on the local resistances is assumed to be equal to $10 \%$ of the linear loss. A preliminary set of diameters that produce a constant specific pressure drop equal to:

$$
\begin{gathered}
\frac{P_{\mathrm{H}}^{2}-P_{\mathrm{K}}^{2}}{1,1 \cdot \Sigma l}=\frac{200^{2}-150^{2}}{1,1 \cdot 360}=\frac{40000-22500}{396}=44,19 \mathrm{kPa} / \mathrm{m} \\
d_{1}=\sqrt[4,75]{\frac{0,0446 \cdot 0,00036 \cdot 0,72 \cdot(750)^{1,75}}{44,19 \cdot 10^{-6}}}=\sqrt[4,75]{\frac{0,0446 \cdot 0,00036 \cdot 0,72 \cdot 107487,3}{44,19 \cdot 10^{-6}}} \\
=8,6 \mathrm{~cm} \\
A=\frac{0,101325}{0,175162 \cdot 3,14^{2}}=0,00036
\end{gathered}
$$

Calculation for the gas distribution network section 2:

$$
\begin{gathered}
R e=0,0354 \cdot \frac{450}{7 \cdot 0,000135}=165571 \\
d_{2}=\sqrt[4,75]{\frac{0,0446 \cdot 0,00036 \cdot 0,72 \cdot(450)^{1,75}}{44,19 \cdot 10^{-6}}}=\sqrt[4,75]{\frac{0,0446 \cdot 0,00036 \cdot 0,72 \cdot 43966,5}{44,19 \cdot 10^{-6}}} \\
=7,16 \mathrm{~cm}
\end{gathered}
$$

Calculation for the gas distribution network section 3:

$$
R e=0,0354 \cdot \frac{200}{5 \cdot 0,000135}=104889
$$




$$
\begin{gathered}
d_{2}=\sqrt[4,75]{\frac{0,0446 \cdot 0,00036 \cdot 0,72 \cdot(200)^{1,75}}{44,19 \cdot 10^{-6}}}=\sqrt[4,75]{\frac{0,0446 \cdot 0,00036 \cdot 0,72 \cdot 10636,59}{44,19 \cdot 10^{-6}}} \\
=5,69 \mathrm{~cm}
\end{gathered}
$$

We determine the pressure loss in the gas distribution network section 1:

$$
\begin{aligned}
& P_{\mathrm{H}}^{2}-P_{\mathrm{K}}^{2}=\frac{P_{0}}{81 \cdot \pi^{2}} \cdot \lambda \cdot \frac{\mathrm{Q}_{0}^{2}}{d^{5}} \cdot \rho_{0} \cdot l=1,2687 \cdot 10^{-4} \cdot 0,015 \cdot \frac{750^{2}}{9^{5}} \cdot 0,73 \cdot 120 \\
& =1,2687 \cdot 10^{-4} \cdot 0,015 \cdot \frac{562500}{59049} \cdot 0,73 \cdot 120=0,001588 \mathrm{MPa}^{2} \\
& =1588 \kappa \Pi a^{2}
\end{aligned}
$$

Determining the Reynolds number:

$$
R e=\frac{Q_{0}}{9 \cdot \pi \cdot d \cdot \vartheta}=0,0354 \cdot \frac{750}{9 \cdot 0,000135}=218518
$$

We determine the hydraulic smoothness of the inner wall of the gas pipeline:

$$
\left(\frac{0,0007}{9}\right)=0,00008<23
$$

As the number $\operatorname{Re}>100000$, the coefficient of hydraulic friction $\lambda$ is determined by the formula

$$
\begin{gathered}
\lambda=\frac{1}{(1,82 \cdot \lg R e-1,64)^{2}}=\frac{1}{(1,82 \cdot \lg 218518-1,64)^{2}}=\frac{1}{(1,82 \cdot 5,339-1,64)^{2}} \\
=\frac{1}{8,077^{2}}=0,015
\end{gathered}
$$

We determine the pressure loss in the gas distribution network section 2:

\begin{tabular}{|c|c|c|c|c|c|c|c|c|c|}
\hline \multicolumn{2}{|c|}{ Number } & \multirow{2}{*}{ 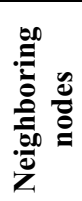 } & \multirow[b]{2}{*}{$\begin{array}{c}\delta \rho \\
\mathbf{k P a}\end{array}$} & \multicolumn{6}{|c|}{ Supporting calculations } \\
\hline$\stackrel{0}{\circ}$ & : & & & $Q^{0.38}$ & $l^{1,19}$ & $\delta p^{-1,19}$ & $\mathbf{A}$ & $A \delta p^{-1,19}$ & $\frac{A \delta^{-1,19}}{\delta p}$ \\
\hline \multirow{4}{*}{$\mathrm{I}$} & 1 & - & -1589 & 12,37 & 298 & & 3686,26 & $-0,55$ & 0,00035 \\
\hline & 2 & II & 2008 & 10,19 & 298 & 0,00012 & 3036,62 & 0,36 & 0,000179 \\
\hline & - & - & - & - & - & - & - & $-0,19$ & 0,000529 \\
\hline & - & - & - & \multicolumn{6}{|c|}{ error rate $\frac{0,19 \cdot 100}{0,5 \cdot 0,91}=41,76$} \\
\hline \multirow{4}{*}{ II } & 2 & $\mathrm{I}$ & -2008 & 10,19 & 298 & 0,00012 & 3036,62 & $-0,36$ & 0,000179 \\
\hline & 3 & - & 2133,8 & 7,49 & 298 & 0,00011 & 2232,02 & 0,25 & 0,00012 \\
\hline & - & - & - & - & - & - & - & $-0,14$ & 0,000299 \\
\hline & - & - & - & \multicolumn{6}{|c|}{ error rate $\frac{0,14 \cdot 100}{0,5 \cdot 0,6}=45,9$} \\
\hline
\end{tabular}

$$
\begin{aligned}
P_{\mathrm{H}}^{2}-P_{\mathrm{K}}^{2}= & \frac{P_{0}}{81 \cdot \pi^{2}} \cdot \lambda \cdot \frac{\mathrm{Q}_{0}^{2}}{d^{5}} \cdot \rho_{0} \cdot l=1,2687 \cdot 10^{-4} \cdot 0,015 \cdot \frac{450^{2}}{7^{5}} \cdot 0,73 \cdot 120 \\
& =0,00200858 \mathrm{MPa}^{2}=2008 \mathrm{kPa}^{2}
\end{aligned}
$$

We determine the pressure loss in the gas distribution network section 3:

$P_{\mathrm{H}}^{2}-P_{\mathrm{K}}^{2}=1,2687 \cdot 10^{-4} \cdot 0,015 \cdot \frac{200^{2}}{5^{5}} \cdot 0,73 \cdot 120=0,0021338 \mathrm{M \Pi а}^{2}=2133,8 \mathrm{kPa}^{2}$

The resulting value of the final pressure in front of the subscriber:

$$
\begin{gathered}
P_{\mathrm{K}}^{2}=\sqrt{P_{\mathrm{H}}^{2}-\Sigma \delta p}=\sqrt{40000-(1588+2008,8+2133,8)}=\sqrt{40000-5730,6} \\
=185,1 \mathrm{kPa}
\end{gathered}
$$

We calculate the nodal correction pressures $\Delta \mathrm{p}$ and the optimal pressure differences in the sections. The results of the calculations are summarized in Table 2.

Table 2. Determination of optimal pressure differences in the sections - supporting calculations. 
Next, we will consistently calculate the correction pressure losses for the sections of the gas distribution network. Let's perform 2 iterations.

Below in the text, we will calculate the first correction, and the results of the calculation will be summarized in Table 3:

$$
\begin{gathered}
\Delta p_{\mathrm{I}}^{\prime}=\frac{\sum A_{i} \delta_{A}^{-1,19}}{1,19 \sum \frac{A \delta_{p}^{-1,19}}{\delta p_{i}}=\frac{-0,19}{1,19 \cdot 0,000529}=-301,8} \\
\Delta p_{\mathrm{II}}^{\prime}=\frac{-0,14}{1,19 \cdot 0,000299}=-393,47 \\
\Delta p_{\mathrm{I}}^{\prime \prime}=\frac{\Sigma \Delta p_{0 \mathrm{y}}^{I} \cdot\left(\frac{A \delta^{-1,19}}{\delta p}\right)}{\sum \frac{A \delta_{p}^{-1,19}}{\delta p_{i}}}=\frac{-393,47 \cdot 0,000179}{0,000529}=-133,14 \\
\Delta p_{\mathrm{II}}^{\prime \prime}=\frac{-301,8 \cdot 0,000179}{0,000299}=-180,68 \\
\Delta p_{\mathrm{I}}=\Delta p_{\mathrm{I}}^{\prime}+\Delta p_{\mathrm{I}}^{\prime \prime}=-301,8-133,14=-434,94 \\
\Delta p_{\mathrm{II}}=\Delta p_{\mathrm{II}}^{\prime}+\Delta p_{\mathrm{II}}^{\prime \prime}=-393,47-180,68=-574,15
\end{gathered}
$$

\begin{tabular}{|c|c|c|c|c|c|c|c|c|c|}
\hline \multicolumn{2}{|c|}{ Number } & \multirow{2}{*}{ 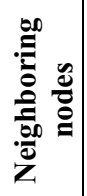 } & \multirow[b]{2}{*}{$\begin{array}{c}\delta \rho \\
\mathbf{k P a}\end{array}$} & \multicolumn{6}{|c|}{ first correction } \\
\hline 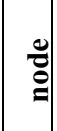 & : & & & $\begin{array}{c}\Delta \rho \\
\mathbf{k P a}\end{array}$ & $\begin{array}{c}\Delta \rho \\
\text { sec- } \\
\text { tion }\end{array}$ & $\delta \rho$ & $\delta p^{-1,19}$ & $(A \delta p)^{-1,19}$ & $\frac{(A \delta p)^{-1,19}}{\delta p}$ \\
\hline \multirow{4}{*}{$\mathrm{I}$} & 1 & - & -1589 & $-434,94$ & $\begin{array}{c}- \\
434,9 \\
4 \\
\end{array}$ & $-2022,9$ & 0,00012 & $-0,442$ & 0,00022 \\
\hline & 2 & II & 2008 & & $\begin{array}{c}139,2 \\
1\end{array}$ & 2147,2 & 0,00011 & 0,334 & 0,00016 \\
\hline & - & - & - & - & - & - & - & $-0,108$ & 0,00038 \\
\hline & - & - & - & \multicolumn{6}{|c|}{ error rate $\frac{0,108 \cdot 100}{0,5 \cdot 0,776}=27$} \\
\hline \multirow{4}{*}{ II } & 2 & I & -2008 & $-574,15$ & $\begin{array}{c}- \\
139 \\
21 \\
\end{array}$ & $-2147,2$ & 0,00011 & $-0,334$ & 0,00016 \\
\hline & 3 & - & 2133,8 & - & $\begin{array}{c}- \\
574 \\
15 \\
\end{array}$ & 1559,65 & 0,00016 & 0,357 & 0,00023 \\
\hline & - & - & - & - & - & - & - & 0,023 & 0,00023 \\
\hline & - & - & - & \multicolumn{6}{|c|}{ error rate $\frac{0,023 \cdot 100}{0,5 \cdot 0,691}=6,65$} \\
\hline
\end{tabular}

Table 3. Determination of optimal pressure differences in the sections - first correction.

Below in the text we will calculate the second correction and the results of the calculation will be summarized in Table 4:

$$
\begin{gathered}
\Delta p_{\mathrm{I}}^{\prime}=\frac{\sum A_{i} \delta_{A}^{-1,19}}{1,19 \sum \frac{A \delta_{p}^{-1,19}}{\delta p_{i}}}=\frac{-0,108}{1,19 \cdot 0,00038}=-238,8 \\
\Delta p_{\mathrm{II}}^{\prime}=\frac{-0,23}{1,19 \cdot 0,00023}=84
\end{gathered}
$$




$$
\begin{gathered}
\Delta p_{\mathrm{I}}^{\prime \prime}=\frac{\Sigma \Delta p_{0 \mathrm{y}}^{I} \cdot\left(\frac{A \delta^{-1,19}}{\delta p}\right)}{\sum \frac{A \delta_{p}^{-1,19}}{\delta p_{i}}}=\frac{84 \cdot 0,00016}{0,00038}=35,37 \\
\Delta p_{\mathrm{II}}^{\prime \prime}=\frac{-38,8 \cdot 0,00016}{0,00023}=-166,1 \\
\Delta p_{\mathrm{I}}=-238,8+35,37=-203,43 \\
\Delta p_{\mathrm{II}}=84-166,1=-82,1
\end{gathered}
$$

\begin{tabular}{|c|c|c|c|c|c|c|c|c|c|}
\hline \multicolumn{2}{|c|}{ Number } & \multirow{2}{*}{ 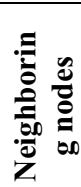 } & \multirow[b]{2}{*}{$\begin{array}{c}\delta \rho \\
\mathbf{k P a}\end{array}$} & \multicolumn{6}{|c|}{ second correction } \\
\hline$\stackrel{\check{g}}{\Xi}$ & : & & & $\begin{array}{c}\Delta \rho \\
\text { node }\end{array}$ & $\begin{array}{c}\Delta \rho \\
\text { sec- } \\
\text { tion }\end{array}$ & $\delta \rho$ & $\delta p^{-1,19}$ & $A \delta^{-1,19}$ & - \\
\hline \multirow{4}{*}{ I } & 1 & - & -1589 & & $-203,43$ & 2226,3 & 0,0001 & 0,37 & - \\
\hline & 2 & II & 2008 & $-203,43$ & $-121,33$ & 2025,4 & 0,00012 & 0,35 & - \\
\hline & - & - & - & - & - & - & - & - & - \\
\hline & - & - & - & \multicolumn{6}{|c|}{ error rate $\frac{0,01 \cdot 100}{0,5 \cdot 0,73}=2,7$} \\
\hline \multirow{4}{*}{ II } & 2 & I & -2008 & $-82,1$ & $\begin{array}{c}121, \\
33\end{array}$ & 2025,4 & 0,00012 & 0,36 & - \\
\hline & 3 & - & 2133,8 & & $-82,1$ & 1477,6 & 0,00017 & 0,38 & - \\
\hline & - & - & - & - & - & - & - & 0,02 & - \\
\hline & - & - & - & \multicolumn{6}{|c|}{ error rate $\frac{0,02 \cdot 100}{05071}=1,4$} \\
\hline
\end{tabular}

Table 4. Determination of optimal pressure differences in the sections - second correction.

The additional pressure to the difference in the section in the absence of a neighboring node is equal to $\Delta \mathrm{p}$, and in the presence of a neighboring node is equal to $\Delta \mathrm{p} "+" \Delta \mathrm{p}$ of the neighboring node with the opposite sign.

$$
\begin{gathered}
\Delta p_{1}=-203,43 \\
\Delta p_{2}=-203,43+82,1=121,33
\end{gathered}
$$

The first fix reduced the error of the nodal equations to $27 \%$ and $6.65 \%$. The error for the first node is significant, so we perform a second correction. The calculations for the second correction are similar to the first one. As a result of the second correction, the errors become equal to $2.7 \%$ and $1.4 \%$.

From the table. 1 it follows that the difference in section 1 increases from $1588 \mathrm{kPa}$ to $2226.3 \mathrm{kPa}$, which leads to a decrease in diameter, and the difference in section 2 increases slightly, the pressure difference from $2008 \mathrm{kPa}$ to $2025.9 \mathrm{kPa}$. In this case, it is impossible to meet this requirement, since the adjacent diameters do not differ much from each other. The gas drop in section 3 is significantly different from $2133.8 \mathrm{kPa}$ to $1447.6 \mathrm{kPa}$, so it is possible to provide for the possibility of increasing the diameter.

Now we determine the values of the section diameters at the new gas pressure drops for:

- gas distribution network section 1:

$$
\begin{gathered}
P_{\mathrm{H}}^{2}-P_{\mathrm{K}}^{2}=\frac{P_{0}}{81 \cdot \pi^{2}} \cdot \lambda \cdot \frac{\mathrm{Q}_{0}^{2}}{d^{5}} \cdot \rho_{0} \cdot l \\
0,0022263=1,2687 \cdot 10^{-4} \cdot 0,015 \cdot \frac{750^{2}}{d^{5}} \cdot 0,73 \cdot 120 \\
0,0022263=1,2687 \cdot 10^{-4} \cdot 0,015 \cdot \frac{562500}{d^{5}} \cdot 0,73 \cdot 120 \\
0,0022263=\frac{93,77}{d^{5}}
\end{gathered}
$$


- gas distribution network section 2:

$$
\begin{array}{r}
d^{5}=42119,2 \\
d=8,4 \mathrm{~cm}
\end{array}
$$

$$
\begin{gathered}
0,002025=1,2687 \cdot 10^{-4} \cdot 0,015 \cdot \frac{450^{2}}{d^{5}} \cdot 0,73 \cdot 120 \\
0,002025=1,2687 \cdot 10^{-4} \cdot 0,015 \cdot \frac{202500}{d^{5}} \cdot 0,73 \cdot 120 \\
0,002025=\frac{33,758}{d^{5}} \\
d^{5}=16670,7 \\
d=6,9 \mathrm{~cm}
\end{gathered}
$$

- gas distribution network section 3:

$$
\begin{gathered}
0,001477=1,2687 \cdot 10^{-4} \cdot 0,015 \cdot \frac{200^{2}}{d^{5}} \cdot 0,73 \cdot 120 \\
0,001477=1,2687 \cdot 10^{-4} \cdot 0,015 \cdot \frac{40000}{d^{5}} \cdot 0,73 \cdot 120 \\
0,001477=\frac{6,668}{d^{5}} \\
d^{5}=4514,75 \\
d=5,38 \mathrm{~cm}
\end{gathered}
$$

\section{Conclusions}

Let's compare the savings obtained by comparing the selected diameters for the initial and final distribution of the pressure drop across the network sections.

We will calculate the material characteristics of the network at a given constant total pressure drop in the network, but with a modified redistribution of the pressure drop across the network sections

$$
\begin{aligned}
& M_{1}=\frac{1}{1000}(90 \cdot 120+70 \cdot 120+50 \cdot 120)=25.2 \mathrm{~m} \cdot \mathrm{m} \\
& M_{2}=\frac{1}{1000}(84 \cdot 120+69 \cdot 120+54 \cdot 120)=24.84 \mathrm{~m} \cdot \mathrm{m}
\end{aligned}
$$

Thus, there is a decrease in the material characteristics of the gas distribution network by $0.36 \mathrm{~m} * \mathrm{~m}$, that is, by $1.43 \%$.

The results of the calculations allowed us to determine the optimal piezometer, which corresponds to the minimum cost of the gas distribution system of medium and high pressure gas networks. The calculation of the nodal correction pressures and pressure differences in the sections was performed. The calculations made have allowed to obtain metal savings of $1,43 \%$.

\section{References}

1. Russia's Energy Strategy for the Period up to 2035. URL: http://www.energystrategy.ru/ (access date: 02.09.2018).

2. A.A. Ionin Gas supply. Moscow (1989)

3. V.A. Zhila Gas supply: textbook for university students in the specialty "Heat and Gas Supply and Ventilation". Moscow (2014)

4. A.V. Laktyun'kin Selection of the degree of ringing of the high-pressure gas distribution network for the medium-sized settlement. (Moscow, 2018) 
5. A.K. Klochko Development of the concept of rational design of gas distribution networks by the iterative search method: (Ph.D. thesis in Engineering Science. Moscow, 2012)

6. Gradient methods. URL: https://studfiles.net/preview/960965/(access date: 01.09.2018).

7. V.A. Zhila, A.K. Klochko, E.A. Gusarova Internet Bulletin of Volgograd State University of Architecture and Civil Engineering 3 (23), (2012)

8. R.G. Arutjunjan Housing construction 11 (2000)

9. K.A. Kuspekov. Omsk scientific bulletin 1 (107), 14-16 (2012)

10. D.A. Ejbozhenko Approximate methods of the Steiner task solution on the oriented columns. (Ph.D. thesis in physical and mathematical sciences St. Petersburg, 2012)

11. M.A. Bagov, V.C. Kudaev International academy of Sciences. 4, 9-14 (2014)

12. V.N. Melkumov, S.V. Chujkin, A.M. Papshickij, K.A. Skljarov Scientific bulletin of the Voronezh state architectural and construction university. Construction and architecture. 2 (38), 41-48 (2015)

13. V.S. Tarasjan, D.O. Ten. Innovative transport 3 (9),29-32 (2013)

14. V.A. Litvinenko, S.A. Hovanskov, V.S. Hovanskova, E.V. Litvinenko. Informatics, computer facilities and engineering education. 4 (28), 9-16 (2016).

15. K.S. Voronin, Je.D. Shabakaeva, D.D. Shabakaev. Problems of functioning of systems of transport materials of the international scientific and practical conference of students, graduate students and young scientists (with the international participation): in 2 volumes. 18-21 (2016)

16. R. G. Arutyunyan Determination of rational strengthening of the reinforced concrete structures, working in the conditions of seismic impacts, by search optimization method. (Ph.D. thesis in Engineering Science. Moscow, 2000)

17. Yu. A. Tabunshchikov, D. V. Koptev, V.A. Zhila, A.K. Klochko, E. B. Soloveva Gas distribution systems efficiency preference / Scientific and technical magazine Vestnik MGSU 8, 222 (2011).

18. K.M. Adeney, M.J. Korenberg. Neural Networks 13/7, 787-799 (2000).

19. C.-Y. Lin, J.-J. Wang.Transactions on Signal Processing. 1, 363-373 (2014).

20. Y. Masatlioglu, D. Nakajima. Theoretical Economics. 3, 701-728 (2013).

21. A. Oddi, A. Cesta, N. Policella, Smith S.F. Journal of Intelligent Manufacturing 1, 1730 (2010).

22. U. Lechowicz, A. Pollak, A. Podgorska, P. Stawinski, M. Oldak, T. Gambin, A. Franke, B.-S. Petersen, M. Firczuk, H. Skarzynski, R. Ploski.Scientific Reports 1, 2543 (2017)

23. R.Zhang, L. Hanzo. IEEE Vehicular Technology Conference Ottawa (2010)

24. T.H. Lin, J.J. Lin, W.J. Peng, J.H. Liu. Computers \& Chemistry. 2, 109-119 (1999) 